\title{
A Denoising Method Based on Analysis K-SVD and Disagreement Segment and Its Application on EMI Signal
}

\author{
Wen-Ru Gong, Hong-Yi Li, Di Zhao \\ LMIB, School of Mathematics and Systems Science, Beihang University, Beijing 100191, P. R. China \\ E-mail: zdhyl2010@163.com
}

\begin{abstract}
Sparse representation plays an important role in signal processing. Recently, the analysis sparse representation has been attracting more and more attention. In this paper, an improved analysis K-SVD denoising algorithm based on disagreement-segment is proposed. A signal is first divided into small redundant segments, and then the signal can be denoised by the analysis K-SVD algorithm. Considering the gap between the local processing and the global signal recovery, we define a disagreement-segment as the difference between the intermediate locally denoised segment and its corresponding part in the final denoised signal. By adding the disagreementsegment to the analysis K-SVD algorithm, the denoising effect of the analysis K-SVD algorithm has a significant improvement. In addition, the experimental results show that the proposed method outperforms the analysis K-SVD algorithm and other advanced methods.
\end{abstract}

\section{Keywords-analysis K-SVD; EMI signal; disagreement- segment}

\section{INTRODUCTION}

In the last two decades, sparse representation is one of the most widely discussed fields. It has successfully extended from theoretical research to a variety of applications, such as signal classification [1], image and signal denoising [2,31-34], blind sources separation [3] and so on. So far, most denosing literatures about sparse representation are image denoising [4]-[6], and signal denoising is rarely studied. With the rapid development of science and technology, signals is interfered seriously during the transmitting. Therefore, the denoising is an essential procedure in the signal processing.

Signal denoising methods mainly contain the Fourier Transform (FT) [7], Wavelet Threshold Denoising (WTD) [8], Empirical Mode Decomposition (EMD) [9] and other variant methods. FT processes a signal in time-frequency domain, which is merely suitable for stable signal. WTD decomposes a signal into some wavelet basis functions, and an appropriate threshold is set to each basis function. Nevertheless, it is difficult to choose appropriate basic functions and thresholds. EMD method decomposes a signal into a series of intrinsic mode functions (IMFs) according to different time scales, which is especially suitable for the nonlinear and non-stationary signals. Generally, EMD denoising discards high-frequency IMFs directly and remains the rest of IMFs to reconstruct the signal. Numerous improved EMD algorithms have been presented to solve signal denoising problem [10]-[12].

Sparse representation have been widely applied to process various signals, for instance, speech signal, medical signal, radar signal, etc [13]-[15]. Some denoising models based on sparse representation have been constructed, such as NLM [16], K-SVD [17], LSSC [18]. These models are used for image denoising, however, signal denoising model on the basis of sparse representation has rarely been investigated. In recent years, the analysis sparse model has been drawing attention [19]. In this model, the corresponding analysis representation is obtained by the inner product of a signal and the analysis dictionary. The study of the analysis sparse model is still infancy and relevant literatures are few. There are two methods to solve the analysis dictionary: structured dictionary and learning dictionary. The expression of structured dictionary is single and this dictionary lacks self-adaption. Hence, a majority of methods employ dictionary learning algorithm. The well-known analysis dictionary learning algorithm is the analysis K-SVD algorithm (AK-SVD) which has been found enormous potential in denoising field [20].

The analysis K-SVD algorithm was presented in [21]. In image processing, firstly, an image is broken into overlapping patches. And then each image patch is denoised by the analysis K-SVD algorithm. Finally, all de-noised image patches are averaged to reconstruct the image. It should be noted that this method ignores the inter-relations of these patches, which causes the loss of local feature and an undesirable denoising result. In order to overcome this disadvantage, we define disagreement-segment as a way to enhance the relationship among these redundant segments, which reduces the local-global error and generates an improvement for analysis K-SVD algorithm. A signal denoising method based on the K-SVD dictionary algorithm was proposed in [14]. Similar to the method presented in [21], a noisy EEG signal is divided into enough redundant segments and these segments are trained by the K-SVD algorithm. The analysis K-SVD method is still room for improvement in [19]. Therefore, we replace the K-SVD algorithm with the analysis K-SVD algorithm to train dictionary and the corresponding denoising model is also transformed into the analysis sparse model presented in [17]. The image reconstruction method in [22] just averaged all the image patches, which overlooks the boundary error during the partition. To further improve this method, [23] eliminated the boundary error via maximum a posteriori probability (MAP) estimator.

In this paper, we propose an improved analysis K-SVD algorithm based on the disagreement-segment. A Noisy signal is divided into enough overlapping segments so as to use the analysis K-SVD algorithm. These segments can be regarded as training signal set and train it to obtain the corresponding analysis dictionary which has local features of 
noisy signal. In order to reduce the error caused by signal division. We add the notion of disagreement-segment to the analysis K-SVD algorithm to narrow local-global gap, which yields an improvement to analysis K-SVD algorithm. Numerical results indicate that signal to noise ratio (SNR) of the proposed method is larger than the analysis K-SVD denoising. The main contributions of this paper are in the following three aspects. 1) The analysis dictionary from redundant segments can represent the noisy signal simply, all-sidedly, self-adaptively. 2) We define a disagreementsegment concept and add it to the analysis KSVD algorithm, which narrows the gap of the local-global and enhances the effectiveness of denoising method. 3) Experimental results on simulated and real signals demonstrate the validity of the proposed method.

The structure of this paper is organized as follows. In section 2, the analysis K-SVD algorithm is reviewed and the notion of segment-disagreement is described. In section 3 , the proposed denoising method and its solution is given. In section 4, the experimental results on simulated and real data indicate that the proposed method outperforms the analysis K-SVD denoising.

\section{RECENT WORKS}

\section{A. The Analysis K-SVD Denoising}

For a given signal $\mathrm{x} \in R^{n}$ and the analysis dictionary $\Omega \in$ $R^{p \times n}(p \geq n)$, the sparse analysis model is expressed as $\Omega x=h$, where $h \in R^{p}$ is the corresponding analysis representation and the rows of the analysis dictionary are defined as $\left\{\omega_{j}\right\}_{j=1}^{p}$. The number of the zero elements in the sparse analysis representation $h$ is defined as co-sparsity $l=p-\|h\|_{0}$ and the corresponding index set is defined as co-support $\Lambda$. When applied to denoising problem, the analysis sparse model can be transformed into the following

$$
\begin{array}{ll} 
& \{\hat{\mathrm{x}}, \widehat{\Lambda}\}=\operatorname{argmin}\|x-y\|_{2} \\
\text { s.t. } & \Omega_{\Lambda} x=0 \operatorname{Rank}\left(\Omega_{\Lambda}\right)=n-r,
\end{array}
$$

or

$$
\begin{aligned}
& \quad\{\hat{x}, \hat{\Lambda}\}=\operatorname{argminRank}\left(\Omega_{\Lambda}\right) \\
& \text { s.t. } \Omega_{\Lambda} x=0\|x-y\|_{2} \leq \varepsilon,
\end{aligned}
$$

where $\hat{\mathrm{x}}$ stands for the original signal, and $r$ represents the dimension of the space the signal belongs to. The solution to the above problem is referred to as analysis -pursuit. The existing advanced methods have the Backward-Greedy (BG) and the Optimized-Greedy (OBG) method. BG method adopts a greedy strategy to pursuit the estimated signal, which is similar to Match Pursuit (MP) algorithm. Firstly, the inner products of the signal $x$ and row vectors $\left\{\omega_{j}\right\}_{j=1}^{p}$ are explored. If the inner product is under the given threshold, the row with respect to this inner product will be added to the co-support set $\Lambda$. Subsequently, the signal is projected onto the orthogonal complement space of $\Omega_{\Lambda}$ and this projection will be considered as an updated signal. Then, the inner products of the updated signal and row vectors $\left\{\omega_{j}\right\}_{j=1}^{p}$ are recalculated, and the co-support set $\Lambda$ is updated simultaneously. Finally, repeat the above steps until the constraint is satisfied. It starts to update the analysis dictionary after getting the estimated signal. The problem is written as follows

$$
\begin{array}{ll} 
& {\left[\hat{\Omega}, \hat{X},\left\{\hat{\Lambda}_{i}\right\}_{i=1}^{N}\right]=\operatorname{argmin}\|X-Y\|_{F}^{2}} \\
\text { s.t. } & \Omega_{\Lambda_{i}} X_{i}=0, \forall 1 \leq i \leq N \\
& \operatorname{Rank}\left(\Omega_{\Lambda_{i}}\right)=d-r, \forall 1 \leq i \leq N \\
& \left\|\omega_{\mathrm{j}}\right\|_{2}=1 \forall 1 \leq \mathrm{j} \leq \mathrm{p},
\end{array}
$$

the current updating row is related to those training signals which are orthogonal to this row. The rest of the training signals are no influence on the final result. Therefore, (3) can be transformed into the following

$$
\begin{aligned}
& \left\{\widehat{\omega}_{\mathrm{j}}, \widehat{\mathrm{X}}_{\mathrm{J}}\right\}=\operatorname{argmin}\left\|\mathrm{x}_{\mathrm{J}}-\mathrm{Y}_{\mathrm{J}}\right\|_{\mathrm{F}}^{2} \\
& \text { s.t. } \Omega_{\mathrm{i}} \mathrm{x}_{\mathrm{i}}=\mathrm{o}, \forall \mathrm{i} \in \mathrm{J} \\
& \omega_{\mathrm{j}}^{\mathrm{T}} \mathrm{x}_{\mathrm{J}}=\mathrm{o},\left\|\omega_{\mathrm{j}}\right\|_{2}=1,
\end{aligned}
$$

where the matrix $X_{J}$, whose each column is orthogonal to $\omega_{j}$, is the sub-matrix of $X$, and $Y_{J}$ corresponding to $X_{J}$ is a submatrix of Y. Because (4) is difficult to solve directly, (4) is transformed into the following problem

$$
\widehat{\omega}_{\mathrm{j}}=\operatorname{argmin}\left\|\omega_{\mathrm{j}}^{\mathrm{T}} \mathrm{Y}_{\mathrm{J}}\right\|_{2}^{2} \text { s.t. }\left\|\omega_{\mathrm{j}}\right\|_{2}=1
$$

tailored for this problem, $\omega_{\mathrm{j}}$ is updated by calculating the eigenvector relevant to the minimum singular value of $\mathrm{Y}_{\mathrm{J}}$.

\section{B. Disagreement-Segment}

Patch processing is a popular method in image processing. Most of models are essentially patch-based [25] [26]. The idea is that an image is broken into overlapping image patches and each image patch can be handled separately. The disadvantage is that this method neglects the gap between the local processing and the global restored image. Similar to patch processing, the proposed method divides the signal into redundant segments. Considering the gap between the local processing and the global signal recovery, we define the notion of disagreement- segment and add it to the analysis K-SVD algorithm. The difference $m_{i}$ between the local estimation and the global estimation is called as disagreement-segment, which is defined as

$$
\mathrm{m}_{\mathrm{i}}^{\mathrm{k}}=\hat{\mathrm{x}}_{\mathrm{i}}^{\mathrm{k}}-\mathrm{R}_{\mathrm{i}} \mathrm{x}^{\mathrm{k}}
$$

where $\hat{x}_{i}^{k}$ is $i$-th locally denoised segment, and $R_{i} x^{k}$ is the corresponding part from the global estimated signal, both obtained at the $k$ iteration. The updating segment is obtained by discarding $m_{i}^{k}$ from the input signal $R_{i} y$ so as to push the overlapping segments to share their local information 


$$
\begin{aligned}
n_{i}^{k} & =R_{i} y-m_{i}^{k}=R_{i} y-\left(\widehat{x}_{i}^{k}-R_{i} x^{k}\right) \\
& =R_{i} x^{k \varepsilon}+\left(R_{i} y-\widehat{x}_{i}^{k}\right) \\
& =R_{i} x^{k \varepsilon}+r_{i}^{k}
\end{aligned}
$$

From the above expression, we know that $r_{i}^{k}=R_{i} y-\hat{x}_{i}^{k}$ is the $i$-th segment noise, obtained at the $k$ iteration. It can be inferred that the proposed method aims to recover a segment from the global estimation $x^{k}$, corrupted by the methodnoise $r_{i}^{k}$. Therefore, the disagreement segment reduces the local-global gap.

\section{THE PROPOSED METHOD}

This section is devoted to describing the proposed denoising method and the corresponding solution. Firstly, the noisy signal $y$ is divided into $N$ redundant segments and these segments are arranged in the Hankel matrix $Y$

$$
Y=\left[\begin{array}{cccc}
y_{1} & y_{2} & \cdots & y_{N} \\
y_{2} & y_{3} & \cdots & y_{N+1} \\
\vdots & \vdots & \vdots & \vdots \\
y_{d} & y_{d+1} & \cdots & y_{n}
\end{array}\right]
$$

It is obvious that each segment is different from its adjacent segment only by one sample. Unlike common training signal set, its each column is a redundant segment of the signal $y$ rather than a complete signal. In addition, it can be seen that the given signal $y$ can be described by the first row and the last column. Secondly, the matrix $Y$ is trained via the analysis K-SVD algorithm, thereby attaining $N$ locally estimated segments. Finally, the estimated global signal can be obtained by maximum a posterior estimator (MAP). From the Section 2, we can know that there is some lacking between the local estimated segments and the global denoised signal. Therefore, the disagreement segment is added to the analysis K-SVD algorithm to improve the effectiveness of denoising. In other words, we remove the disagreement-segments from input segments to obtain modified segments, and train these by the analysis K-SVD algorithm. Repeat the above iterative process until the constraint is satisfied. The improved analysis K-SVD algorithm narrows the local-global gap as much as possible. (1) is modified as follows:

$$
\begin{aligned}
& \left\{\hat{x}, \Omega, \hat{x}_{i}\right\}= \\
& \left.\min _{x, \Omega, x_{1}} \lambda\|x-y\|_{2}^{2}+\sum_{i=1}^{N} \mu_{i}\left\|\Omega x_{i}\right\|_{0}+\sum_{i=1}^{N}\left\|x_{i}-R_{i} x\right\|_{2}^{2}, 8\right)
\end{aligned}
$$

in which $x$ is the global denoised signal, $\mathrm{x}_{\mathrm{i}}$ is the ith local estimated segment and $R_{i} x$ is the local segment of $x$. The first term is a tradeoff between the noise signal and the denoised signal, and $\lambda$ is a positive control parameter. The second and third terms are same as the analysis K-SVD algorithm, which are just different in writing. The parameter $\mu_{i}>0$ controls sparsity.

It can be seen that there are three unknowns in (8). The three unknowns are the global estimated signal $x$, the analysis dictionary $\Omega$ and the local denoised segment $x_{i}$, respectively. It is impossible to solve three unknowns simultaneously. Here, we initialize $x$ with y and fix an initial analysis dictionary. (8) is completely decoupled into

$$
\tilde{\mathrm{x}}_{\mathrm{i}}=\min _{\tilde{\mathrm{x}}} \mu_{\mathrm{i}}\left\|\Omega \tilde{\mathrm{x}}_{\mathrm{i}}\right\|+\left\|\tilde{\mathrm{x}}_{\mathrm{i}}-\mathrm{R}_{\mathrm{i}} \mathrm{x}\right\|_{2}^{2}
$$

We apply the analysis K-SVD algorithm with disagreementsegment to calculate the above problem. It is worth noting that the analysis K-SVD algorithm can obtain estimated segments directly. For K-SVD algorithm, estimated segments need to be computed by the inner product between the dictionary and sparse coefficient. Therefore, the analysis K-SVD method is simpler than the K-SVD method. In order to solve the estimated signal $x, x_{i}$ and $\Omega$ are fixed and only $x$ varies

$$
\hat{\mathrm{x}}=\min _{\mathrm{x}} \lambda\|\mathrm{x}-\mathrm{y}\|_{2}^{2}+\sum_{\mathrm{i}=1}^{\mathrm{N}}\left\|\tilde{\mathrm{x}}_{\mathrm{i}}-\mathrm{R}_{\mathrm{i}} \mathrm{x}\right\|_{2}^{2},
$$

$x$ can be estimated by computing the derivative of (10) with respect to $\mathrm{x}$ and set it to zero

$$
\lambda(x-y)+\sum_{i} R_{i}^{T}\left(R_{i} x-\tilde{x}_{i}\right)=0
$$

leads out

$$
\hat{\mathrm{x}}=\left(\lambda \mathrm{I}+\sum_{\mathrm{i}} \mathrm{R}_{\mathrm{i}}^{\mathrm{T}} \mathrm{R}_{\mathrm{i}}\right)^{-1}\left(\lambda \mathrm{y}+\sum_{\mathrm{i}} \mathrm{R}_{\mathrm{i}}^{\mathrm{T}} \tilde{\mathrm{x}}_{\mathrm{i}}\right)
$$

In this equation, $I$ is the identity matrix, $\hat{x}$ is the global denoised signal and $T$ stands for matrix transpose. Note that $\sum_{i} R_{i}^{T} R_{i}$ is diagonal and thus its inverse matrix is easy to compute. The calculation is simpler than what it appears. The improved analysis K-SVD algorithm is summarized in Algorithm1.

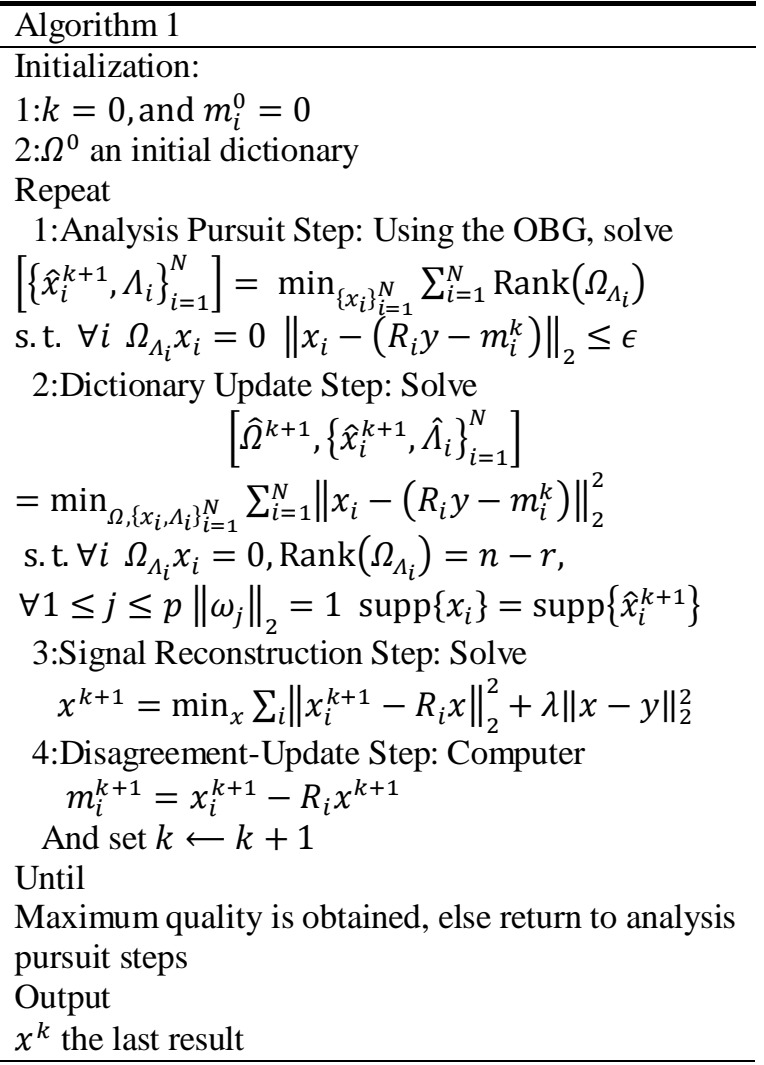




\section{NUMERICAL EXPERIMENT}

In this section, numerical experiments include two parts, simulation data and real data. The experimental results illustrate that the proposed method is superior to analysis KSVD method [21], K-SVD method [27], wavelet softthreshold denoising (WSTD) [28], and EEMD [29].

\section{A. Simulation data}

There are various electromagnetic signals in our Figure1 surrounding. Generally, these signals are non-linear and non-stationary. Here, a generated signal $y(t)$ is composed of aharmonic wave $y_{1}(t)$, two FM signals $y_{2}(t)$ and $y_{3}(t)$, and white Gaussian noise $\mathrm{v}(\mathrm{t})$ with variance 0.5 .

where

$$
y(t)=y_{1}(t)+y_{2}(t)+y_{3}(t)+v(t), t \in[0,1]
$$

$$
\left\{\begin{array}{l}
y_{1}(t)=\cos (7 \pi t) \\
y_{2}(t)=(2+\cos (5 \pi t)) \cos (70 \pi t) \\
y_{3}(t)=\cos (50 \pi t+2 \sin (2 t))
\end{array}\right.
$$

The simulation signal has 1000 sample points. According to the proposed method, the noisy signal is first arranged in Hankel matrix. Here, the dimension of each segment is set to 49 and the size of Hankel matrix is $49 \times 952$, that to say, the number of training samples is 952 . The improved analysis K-SVD method is used to train these training signals. The threshold is set to $1.15 \sqrt{d} \sigma$, where $d$ is the size of segment and $\sigma$ is the noise level. OBG method is used during the analysis pursuit. In dictionary updating stage, the experimental results show that the performance is the best when the rows of the analysis dictionary are 55 . Hence, the size of the analysis dictionary is $55 \times 49$. The initial dictionary is randomly orthogonal to $d-1$ training signals. It can be seen from Fig. 2 that SNR varies with $\lambda$ in the reconstruction. In order to achieve the best performance, parameter $\lambda$ is set to 1.7 and the experiment iterates 20 .
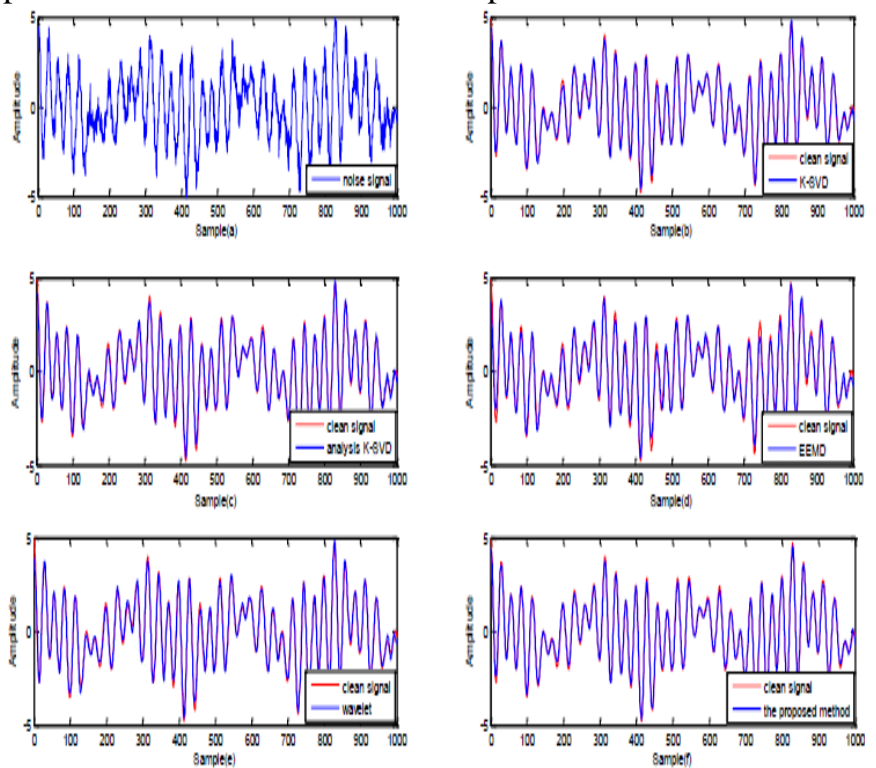

Figure 1. The comparison of different methods.

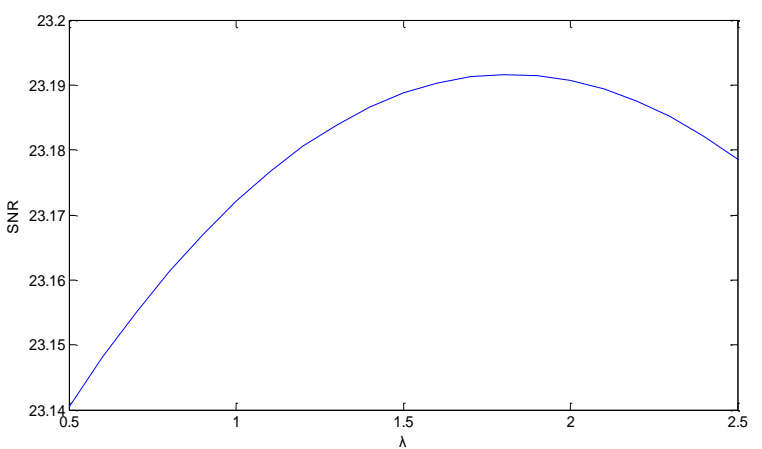

Figure 2. SNR varies with parameter $\lambda$.

The proposed method is compared to K-SVD denoising [27], analysis K-SVD denoising [21], wavelet soft-threshold denoising [28] and EEMD denoising[29]. The Symlets wavelet base is used in the WSTD, and the decomposition layers are set to 5. For EEMD denoising, we discard the first two IMFs which are the high frequency components. The results of different methods are shown in Fig.1, where the red line represents original signal and blue line is denoised signal. As shown in Fig.1, all the methods can remove the noise. However, the proposed method has the best performance. In order to clearly show the results, Fig.3 displays the local difference for various methods. Obviously, the proposed method is closer to the original signal in the peaks and troughs. Thus it has better fitting effect than other methods. The signal-to-noise (SNR) and root-mean-squareerror (RMSE) are to evaluate the quality of the denoising.

$$
\begin{gathered}
\operatorname{SNR}[\mathrm{dB}]=10 \log _{10} \frac{\sum_{\mathrm{n}=1}^{\mathrm{N}} \mathrm{x}^{2}[\mathrm{n}]}{\sum_{\mathrm{i}=1}^{\mathrm{N}}|\mathrm{x}[\mathrm{n}]-\hat{\mathrm{x}}[\mathrm{n}]|^{2}} \\
\operatorname{RMSE}=\frac{1}{\mathrm{~N}} \sum_{\mathrm{n}=1}^{\mathrm{N}}(\mathrm{x}[\mathrm{n}]-\hat{\mathrm{x}}[\mathrm{n}])^{2}
\end{gathered}
$$

where $x[n]$ is the original signal, $\hat{x}[n]$ is denoised signal and $N$ is sample number.

Based on the Table 1, both of K-SVD and analysis KSVD algorithm are superior to wavelet soft threshold. The key point is that the learned analysis dictionary is selfadaptive and local. Although EEMD method is also a selfadaptive method, the effect is poor. Because EEMD discards the first two IMFs, it causes the loss of signal features. It can be seen that the proposed algorithm has maximum SNR and minimum RMSE.

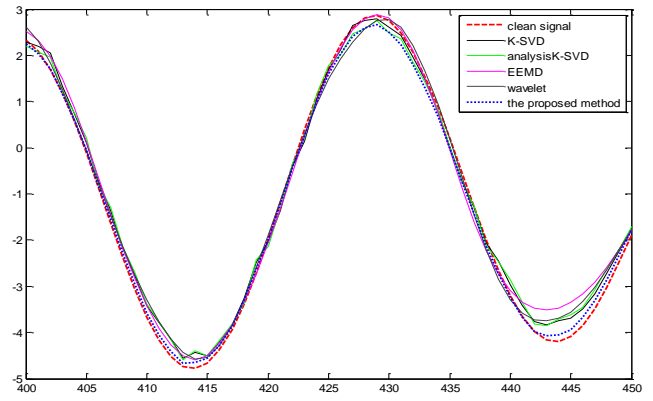

Figure 3. The local comparison of different methods. 
TABLE 1 . DENOISING RESUlTS IN TERMS OF SNR AND RMSE FOR THE COMPARISON APPROACHES

\begin{tabular}{|l|l|l|}
\hline \multicolumn{1}{|c|}{ Method } & \multicolumn{1}{c|}{ SNR } & \multicolumn{1}{c|}{ RMSE } \\
\hline K-SVD[27] & 21.10 & 0.16 \\
\hline Analysis K-SVD[21] & 20.40 & 0.17 \\
\hline EEMD[29] & 16.82 & 0.26 \\
\hline WSTD[28] & 19.38 & 0.19 \\
\hline The proposed method & 23.19 & 0.12 \\
\hline
\end{tabular}

\section{B. Real Data}

Electroencephalogram (EEG) signal is the non-linear and non-stationary electromagnetic signal. In order to prove the effectiveness of the proposed method, we use the EEG signal recorded in [30] for test. We add white Gaussian noise with $\sigma=10$ to the original EEG signal. The noisy EEG signal is arranged in $49 \times 952$ Hankel matrix and the training set is trained by the improved analysis K-SVD algorithm. The settings of analysis pursuit stage are same as the settings of the simulation experiment in analysis pursuit stage. In dictionary updating stage, iteration steps are set to 20 and the rows of the analysis dictionary are 55. It is showed in Fig.4 that the effect is outstanding when parameter $\lambda$ ranges from 1 to 1.5 . Here, we choose $\lambda=1.3$. Figure 6 shows the results on different methods. Different from the simulation experiment, the effect of the wavelet soft threshold denoising method is poor. Nonetheless, the effect of the EEMD denoising is better than the effect of the

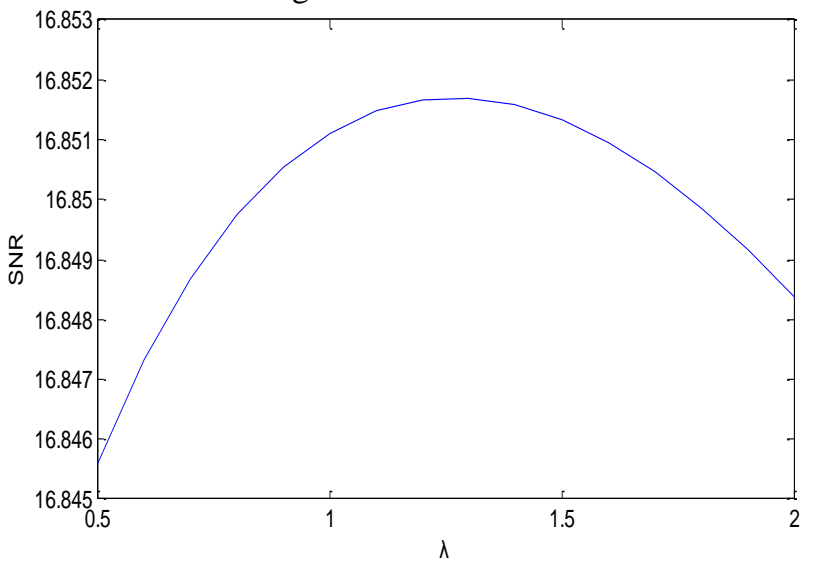

Figure 4. SNR varies with parameter $\lambda$.

wavelet soft threshold denoising. It also states that different methods are suitable for different signals. In addition, it also can be observed from Fig.6 that the proposed method is superior to the analysis K-SVD algorithm, which proofs the validity of adding disagreement-segment to the analysis KSVD algorithm. From Fig.5, the proposed method outperforms comparison methods. Table 2 also theoretically demonstrates the effectiveness of the proposed method.

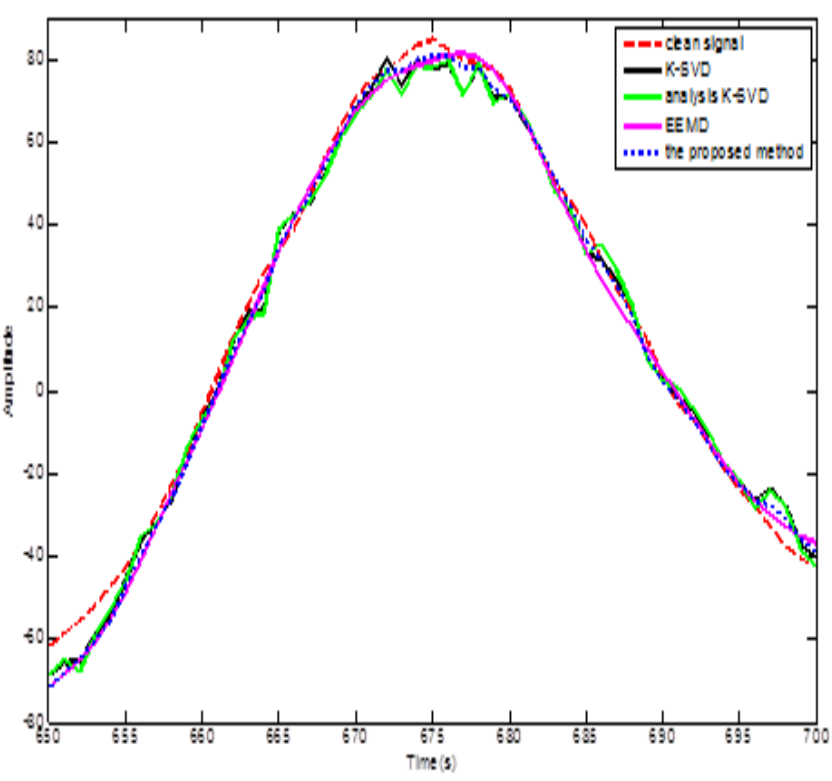

Figure 5. The local comparison of different methods.
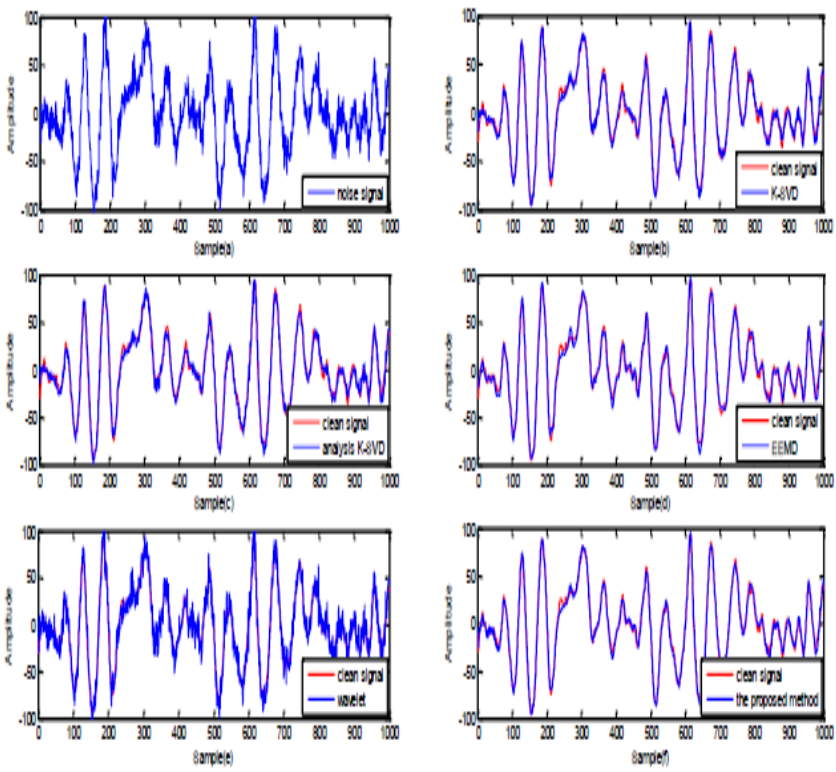

Figure 6. The comparison of different methods.

TABLE 2 DENOISING RESULTS IN TERMS OF SNR AND RMSE FOR THE COMPARISON APPROACHES

\begin{tabular}{|l|l|l|}
\hline \multicolumn{1}{|c|}{ Method } & \multicolumn{1}{c|}{ SNR } & \multicolumn{1}{c|}{ RMSE } \\
\hline K-SVD[27] & 18.13 & 4.74 \\
\hline Analysis K-SVD[21] & 17.20 & 5.27 \\
\hline EEMD[29] & 18.76 & 4.40 \\
\hline WTD[28] & 11.98 & 9.61 \\
\hline The proposed method & 19.70 & 3.95 \\
\hline
\end{tabular}




\section{CONCLUSION}

In this paper, an improved analysis K-SVD is presented. The key is that we defined disagreement-segment. By adding it to the analysis K-SVD algorithm, the analysis K-SVD algorithm is improved effectively. The noisy signal is divided into some overlapping segments which are regarded as training signals. The improved analysis K-SVD algorithm is used to train these signals, and the obtained analysis dictionary has the local features of the noisy signal. Compared with the analysis K-SVD, numerical experimental results prove that the proposed method is excellent.

\section{ACKNOWLEDGEMENT}

This work was supported by the National Natural Science Foundation (Grant no. 61379001).

\section{REFERENCES}

[1] Y. Li, Z. Yu, and N Bi, "Sparse representation for brain signal processing: a tutorial on methods and applications," IEEE Signal Processing Magazine, vol. 31, pp. 96-106, 2014.

[2] E. Michael, A. Michal, "Image denoising via sparse and redundant representations over learned dictionaries," IEEE Transactions on Image Processing, vol. 15, pp.3736-45, 2007.

[3] T. Xu, W. Wang, "A compressed sensing approach for underdetermined blind audio source separation with sparse representation," Statistical Signal Processing, SSP09. IEEE/SP , pp.493-496, 2009.

[4] X. Zhang, X. Feng, and W. Wang, "Image denoising via 2D dictionary learning and adaptive hard thresholding," Pattern Recognition Letters, vol. 34, pp. 2110-2117, 2013.

[5] M. Eksioglu, "Online dictionary learning algorithm with periodic updates and its application to image denoising," Expert Systems with Applications, vol. 41, pp. 3682-3690, 2014.

[6] S. Yang, M. Wang, and M. Wei, "Evolution-enhanced multiscale overcomplete dictionaries learning for image denoising," Engineering Applications of Artificial Intelligence, vol. 25, pp. 1259-1264, 2012.

[7] D. Gabor, "Theory of communication," IEE(London), vol.93, pp. 429-457, 1946.

[8] S. G. Mallat, "Multiresolution representations and wavelets" (January 1, 1988). Dissertations available from Pr Quest. Paper AAI8824767.

[9] N. E. Huang, Z. Shen, and S. R. Long, "The empirical mode decomposition and the Hilbert spectrum for nonlinear and nonstationary time series analysis," Proc. R. Soc. London. vol.Ser. A, 454, pp. 903-995, 1998.

[10] Z. Shang-Yue, L. Yuan-Yuan, and Y. Gong-Liu, "EMD interval thresholding denoising based on correlation coefficient to select relevant modes," Control Conference (CCC), 34th Chinese, IEEE, 2015.

[11] S. A. Elgamel, "EMD thresholding and denoising inspired by wavelet technique," Computer Engineering \& Systems (ICCES), 2014 9th International Conference on. IEEE, 2014.

[12] M. A. Kabir, C. Shahnaz, "Denoising of ECG signals based on noise reduction algorithms in EMD and wavelet domains," Biomedical Signal Processing \& Control, vol. 7, pp. 481-489, 2012.

[13] M. G. Jafari, M.D, Plumbley, "Fast dictionary learning for sparse representations of speech signals[J]," IEEE Journal of Selected Topics in Signal Processing, vol. 5, pp. 1025-1031, 2011.

[14] V. Abolghasemi, S. Ferdowsi, "EEG-fMRI: dictionary learning for removal of ballistocardiogram artifact from EEG," Biomedical Signal Processing \& Control, vol. 18. pp. 186-194, 2015.
[15] M. Li, G. Zhou, and B Zhao, "Radar HRRP adaptive denoising via sparse and redundant representations," Antennas \& Propagation (ISAP), 2013 Proceedings of the International Symposium on, vol. 2013, pp. 1094-1097.

[16] A. Buades, B. Coll, and J. M. Morel, "A review of image denoising algorithms, with a new one," Siam Journal on Multiscale Modeling \& Simulation, vol. 4, pp. 490-530, 2005.

[17] E. Michael, A. Michal, "Image denoising via sparse and redundant representations over learned dictionaries," IEEE Transactions on Image Processing, vpl. 15, pp. 3736-45, 2007.

[18] J. Mairal, F. Bach, and J. Ponce, "Non-local sparse models for image restoration," Computer Vision, 2009 IEEE 12th International Conference on, vol. 2009, pp. 2272-2279, 2009.

[19] S.Nam, M.E.Davies, M. Elad, and R.Gribonval, "The cosparse analysis model and algorithm," submitted to Appl. Comput. Harmon. Anal

[20] T. Peleg, M. Elad, "Performance guarantees of the thresholding algorithm for the cosparse analysis model," IEEE Transactions on Information Theory 59. pp. 1832-1845, 2013.

[21] R. Rubinstein, T. Peleg, and M. Elad, "Analysis K-SVD: A dictionary-learning algorithm for the analysis sparse model," Signal Processing, IEEE Transactions on, vol. 61, pp. 661-677, 2013.

[22] S. Roth, M. J. Black, "Fields of experts: A framework for learning image priors," IEEE Conference on Computer Vision and Pattern Recognition, vol. 2, pp.860-867, June.

[23] O. G. Guleryuz, "Nonlinear approximation based image recovery using adaptive sparse reconstructions and iterated denoising: Part I and Part II," to appear in the IEEE Trans. on Image Processing.

[24] Y. Romano, M. Elad, " Patch-disagreement as away to improve KSVD denoising," Acoustics, Speech and Signal Processing (ICASSP), IEEE, 2015.

[25] R. Idan, E. Michael, and C. Israel, "Image processing using smooth ordering of its patches," IEEE Transactions on Image Processing, vol 22, pp. $2764-2774,2013$

[26] G. Yu, G. Sapiro, and S. Mallat, "Solving inverse problems with piecewise linear estimators: from Gaussian mixture models to structured sparsity," IEEE Transactions on Image Processing A Publication of the IEEE Signal Processing Society, vol. 21, pp. 24812499, 2012.

[27] M. Aharon, M. Elad, and A. Bruckstein, "K-SVD: an algorithm for designing overcomplete dictionaries for sparse representation," Signal Processing, IEEE Transactions on, vol. 54, pp. 4311-4322, 2006.

[28] D. L. Donoho, "De-noising by soft-thresholding," Information Theory IEEE Transactions on, vol. 41, pp. 613-627, 1995.

[29] Z. Wu, N. E. Huang, Ensemble Empirical Mode Decomposition: a noise assisted data analysis method," Adv Adapt Data Analy 1: 1-42, Advances in Adaptive Data Analysis, vol. 1, pp. 1-41, 2009.

[30] R. G. Andrzejak, K. Schindler, and C. Rummel, "Nonrandomness, nonlinear dependence, and nonstationarity of electroencephalographic recordings from epilepsy patients," Physical Review E, vol. 86, 2012.

[31] J. Chen, Y. H. Wang and Y. Y. Tang, "Person Re-Identification by Exploiting Spatio-Temporal Cues and Multi-View Metric Learning," IEEE Signal Processing Letters, vol. 23, no. 7, pp. 998-1002, 2016.

[32] J. Chen, Z. X. Zhang and Y. H. Wang, "Relevance Metric Learning for Person Re-Identification by Exploiting Listwise Similarities," IEEE Trans. on Image Proccessing, vol. 24, no. 12, pp. 4741-4755, 2015

[33] H. Y. Li, L. Li, D. Zhao, J. X. Chen and P. D. Wang, "Reconstruction and basis function construction of electromagnetic interference source signals based on Toeplitz-based singular value decomposition," IET Signal Processing, 10.1049/iet-spr.2016.0307, 2016.

[34] H. Y. Li, D. Zhao, F. Dai and D. L. Su, "On the spectral radius of a nonnegative centrosymmetric matrix," Applied Mathematics and Computation, vol. 218, no. 9, pp. 4962-4966, 2012. 